\title{
Riscos Ocupacionais dos Profissionais de Enfermagem atuantes no SAMU 192
}

\author{
Marta Oliveira Nascimento ${ }^{1}$; Giovana Fernandes Araújo ${ }^{2}$
}

\begin{abstract}
Resumo: Os profissionais atuantes no Samu 192 são suscetíveis a diversos riscos ocupacionais. O objetivo deste artigo foi avaliar a percepção de riscos ocupacionais que os profissionais de enfermagem atuantes no atendimento móvel de urgência. Estudo descritivo com uma abordagem qualitativa, onde participaram quatorze profissionais (enfermeiros e técnico de enfermagem) que trabalham no Atendimento Pré Hospitalar - APH. Os dados foram coletados através de uma entrevista semiestruturada. Constatou-se que os profissionais atuantes no Samu 192 tem conhecimento dos fatores de risco envolvidos durante o seu labor, considerando a sobrecarga de trabalho aliado às falhas na estrutura organizacional, fatores estes determinantes para potencializar os riscos ocupacionais. A identificação dos fatores de riscos os quais estão expostos contribui de forma efetiva para elaboração de medidas de proteção necessárias a fim de minimizar os riscos ocupacionais.
\end{abstract}

Descritores: Samu, Enfermgem, Riscos ocupacionais.

\section{Occupational Risks of Nursing Professionals Working in SAMU 192}

\begin{abstract}
The professionals working on the Samu 192 are susceptible to various occupational hazards. The objective of this article was to evaluate the perception of occupational hazards that the nursing professionals working in emergency mobile service. A descriptive study with a qualitative approach, involving fourteen professionals (nurses and nursing technician) who work in Prehospital Care (APH). Data were collected through a semi-structured interview. It was verified that the professionals working in the Samu 192 are aware of the risk factors involved during their work, considering the work overload associated with the failures in the organizational structure, factors that determine the potential for occupational risks. The identification of the risk factors they are exposed contributes effectively to the elaboration of the necessary protection measures in order to minimize the occupational risks.
\end{abstract}

Descriptors: Samu, Enfermgem, Occupational risks.

\footnotetext{
${ }^{1}$ Discente do $10^{\circ}$ semestre do curso de Enfermagem da Faculdade Independente do Nordeste - FAINOR. Email: martinhaon@ hotmail.com ${ }^{2}$ Enfermeira, Mestre em Meio Ambiente e Sustentabilidade, docente do curso de Enfermagem da Faculdade Independente do Nordeste FAINOR. Email: giovanafaraujo@hotmail.com
} 
Id on Line Revista Multidisciplinar e de Psicologia

Id on Line Multidisciplinary and Psycology Journal

\section{Introdução}

Trabalhar consiste em uma das práticas fundamentais na vida do homem, pois, com ele, o ser humano consegue a sua subsistência e do seu núcleo familiar. Os riscos ocupacionais são considerados acontecimentos em âmbito trabalhista, seja individualmente ou por condições da instituição, que possa gerar algum dano a saúde do trabalhador, provocando a morte ou adoecimento. Portanto, o trabalhador durante as suas atividades, fica exposto aos inúmeros acidentes de trabalho existentes (SILVA; LIMA; MARZIALE, 2012).

Acidente de trabalho é o que ocorre pelo exercício do trabalho a serviço da empresa, ou ainda pelo exercício do trabalho dos segurados especiais, provocando lesão corporal ou perturbação funcional que cause morte, a perda ou redução da capacidade para o trabalho permanente ou temporário (BUDEL, 2012).

As doenças profissionais constituem um grave problema de saúde pública. Segundo a Organização Mundial de Saúde (OMS) e a Organização Internacional do Trabalho (OIT), a globalização contribuiu de forma significativa para que as doenças e acidentes de trabalho aumentasse de forma exponencial, matando ou mutilando mais de 2 milhões de trabalhadores em todo globo (OMS, 2015).

Os profissionais de saúde só foram considerados uma categoria de alto risco para acidentes de trabalho a partir do século XX, quando a ocorrência dos riscos biológicos foi relacionada com as doenças que atingiam especificamente os trabalhadores da área da saúde. $\mathrm{Na}$ área da saúde, os profissionais de enfermagem, especialmente os profissionais do serviço de atendimento pré-hospitalar, estão expostos a diversos riscos de natureza física, biológica, química e psicossocial (DE OLIVEIRA, 2013).

O serviço de Atendimento Pré-Hospitalar- APH destina-se ao atendimento de urgência e emergência nas residências, locais de trabalho e vias públicas. Esse tipo de serviço é muito específico e acontece nos mais diversos ambientes possíveis, sem formas de controle efetivas. Diante disso, os profissionais que atuam nesse segmento estão propensos aos mais distintos tipos de riscos ocupacionais.

Os trabalhadores desse setor estão expostos a riscos peculiares à atividade, como risco biológico (pelo contato com micro-organismos), físico (iluminação, temperatura, ruído, radiações, etc), químico (manipulação de desinfetantes, medicamentos, etc), psicossocial 
Id on Line Revista Multidisciplinar e de Psicologia

Id on Line Multidisciplinary and Psycology Journal

(atenção constante, pressão da chefia, estresse e fadiga, ritmo acelerado, trabalho em turnos alternados, etc) e ergonômico (peso excessivo, trabalho em posições incômodas) (ZAPPAROLI; MARZIALE, 2006; BRASIL, 2014).

O profissional do APH atua em ambientes diversos, lidando com situações inusitadas que podem colocar sua vida em risco. Tal risco que a equipe de APH está exposta é devido aos inúmeros obstáculos que aparecem durante a sua atuação profissional, tais como: falha na qualificação técnica ou científica, treinamento inadequado, situações de difícil acesso, falta de segurança na cena, espaço diminuído para realizar procedimentos e manobras, tanto com o veículo parado como em movimento, e falta de protocolos exclusivos para prevenção e controle de infecção (ARAUJO; MOREIRA, 2015).

No entanto, conforme Graça Júnior et al. (2009), a identificação precoce dos riscos ocupacionais a que a equipe de enfermagem está exposta contribui efetivamente na prevenção e no controle dos riscos e dos acidentes de trabalho, reduzindo os danos à saúde do trabalhador e os prejuízos à instituição.

Diante disso, este estudo se justifica pela necessidade de discutir os riscos em que os profissionais de enfermagem, no APH, estão expostos durante a sua rotina laboral. E, a partir daí despertar reflexões e estratégias para a minimização dos mesmos. Nesta perspectiva, a presente pesquisa tem como objetivo geral: avaliar a percepção de riscos ocupacionais que os profissionais de enfermagem atuantes no atendimento móvel de urgência. E, como objetivos específicos: traçar o perfil sócio cultural dos participantes; verificar as situações que configuram maior risco à integridade psicofísica dos profissionais atuantes no SAMU, segundo sua percepção; e identificar as estratégias utilizadas pelos profissionais para minimizar os riscos aos quais estão expostos.

\section{Metodologia}

Trata-se de uma pesquisa descritiva com uma abordagem qualitativa. Constituiu a amostra do estudo 14 profissionais de enfermagem do atendimento às urgências extra hospitalares, SAMU 192, sendo 10 técnicos de enfermagem e 4 enfermeiros, de uma cidade de médio porte no sudoeste da Bahia. Este serviço possui 5 enfermeiros e 35 técnicos de 
Id on Line Revista Multidisciplinar e de Psicologia

Id on Line Multidisciplinary and Psycology Journal

enfermagem. $\mathrm{O}$ fechamento amostral ocorreu no momento em que houve uma saturação teórica emitida nas falas dos informantes, ou seja, no momento em que os dados apresentaram certa repetição (FONTANELLA; RICAS; TURATO,2008 ).

Antes da realização da pesquisa, esta foi submetida ao Comitê de Ética da Faculdade Independente do Nordeste (CEP- FAINOR), de acordo com as normas éticas da Resolução ${ }^{\circ}$ 466/12 do Conselho Nacional de Saúde (CNS). Após parecer favorável, CAE 59235216.8.0000.5578, foi iniciada a coleta de dados.

A coleta de dados foi obtida através de entrevista gravada utilizando-se um roteiro semiestruturado elaborado e aplicado pela pesquisadora, no período de 19 a 24 de novembro de 2016. As entrevistas foram realizadas individualmente, no local de trabalho (Base do SAMU), após os participantes assinarem o Termo de Consentimento Livre e Esclarecido TCLE, considerando as determinações da Resolução 466/2012 do Conselho Nacional de Saúde. Após a realização das entrevistas, estas foram transcritas para o programa Word/Starter 2010.

Os dados foram analisados visando a identificação das categorias e unidades temáticas a partir da utilização do referencial de Bardin. Os dados foram operacionalizadas a partir das seguintes ações: leitura flutuante e aprofunda dos dados, grifando os relatos significativos, semelhantes e diferentes; recortes de fragmentos grifados; busca de categorias a partir das temáticas mencionadas no objetivo; elaboração de uma lista de fala dos entrevistados por categorias; escolha das falas que possuem maior poder de síntese e abrangência, informações dentre os conteúdos das entrevistas para exemplificar as categorias emergentes. O discurso dos entrevistados foi identificado com a sigla S, seguido de numeração de 1 a 14 .

\section{Resultados e Discussões}

O estudo foi realizado com 14 sujeitos pertencentes às seguintes categorias: 04 enfermeiros e 10 técnicos de enfermagem. 08 são do gênero feminino e 06 do gênero masculino, a faixa etária variou entre 30 e 45 anos. Em relação ao período que trabalhavam na instituição o tempo variou entre 04 a 13 anos. A partir da análise das respostas emergiram duas categorias, a saber: O conceito de riscos ocupacionais e os riscos presentes no ambiente de trabalho da equipe e as medidas de prevenção de acidentes e adoecimento utilizados no ambiente de trabalho. 
Id on Line Revista Multidisciplinar e de Psicologia

Id on Line Multidisciplinary and Psycology Journal

\section{Categoria 01: $O$ conceito de riscos ocupacionais e os riscos presentes no ambiente de trabalho da equipe}

A grande maioria dos profissionais de enfermagem considera como riscos ocupacionais todos os riscos e danos que incidem sobre a saúde humana em relação a seu ambiente de trabalho, conforme os discursos:

\footnotetext{
"A gente entende os riscos ocupacionais como todos aqueles riscos relacionados ao trabalho que expõe o trabalhador a desenvolver doenças ocupacionais” (S2).

"Acho que existe risco ocupacional quando há possibilidade de um profissional sofrer um determinado dano em seu ambiente de trabalho" (S4).

"Para mim o risco ocupacional são aqueles riscos que o trabalhador está exposto devido à situação ou tipo de trabalho que desenvolve. São riscos a integridade física, emocional ou outras situações que podem comprometer a saúde do trabalhador" (S10).

"São riscos que podem trazer maleficios e problemas a saúde da gente, podendo deixar traumas permanentes" (S13).

"Acho que se resume em todos os perigos inerentes aos trabalhadores em seu ambiente de trabalho em decorrência de suas atividades laborais, sem as devidas orientações elou precauções cabiveis" (S11).
}

Os profissionais do APH atuam em cenários diversos, como ambiente hospitalar, domiciliar ou atendimento móvel, estando cotidianamente expostos aos riscos ocupacionais. Os riscos ocupacionais são considerados como uma ou mais condições de trabalho com o potencial para causar danos nocivos ao organismo, resultando em um desequilíbrio físico, social e mental dos trabalhadores. A legislação brasileira regulamenta a saúde do trabalhador, a existência de riscos ocupacionais e o reconhecimento destes no processo da atividade profissional (ALMEIDA, 2012).

A constante exposição dos profissionais do APH aos riscos ocupacionais associa a possibilidade deste profissional de sofrer algum tipo de acidente ou adquirir doenças e agravos de origem ocupacional (NEVES et. al, 2011).

Identificar os riscos aos quais estes profissionais estão expostos tem como objetivos principais favorecer o reconhecimento e avalia-los, buscando monitorar, gerenciar e se possível, diminuir a incidência de acidentes de trabalho durante o desenvolvimento de suas atividades laborais. Foi possível inferir, através das falas dos entrevistados, que os mesmos reconhecem os riscos aos quais estão expostos em seu ambiente de trabalho: 
Id on Line Revista Multidisciplinar e de Psicologia

Id on Line Multidisciplinary and Psycology Journal

"Riscos químicos, físicos e biológicos". (S1)

"Riscos psicológicos, químicos, biológicos, ergonômicos e ambientais" (S2 - S3).

"Riscos físicos, químicos, biológicos, ergonômicos, radiológicos (radioativos) e riscos de acidentes". (S11).

"No meu trabalho, tenho risco físico (ruidos), biológicos (sangue, tecidos, fungos, bactérias) e ergonômicos” (S12).

Segundo o Ministério do Trabalho e Emprego, os riscos ocupacionais são classificados na Norma Regulamentadora 09 (NR) como riscos físicos, químicos e biológicos e na NR 5, em seu Anexo IV sobre o mapa de riscos, trata da ocorrência dos riscos ergonômicos e de acidentes. Os riscos são divididos em cinco grupos: os riscos físicos são classificados por umidade, calor e frio extremo, vibrações, radiações ionizantes e não ionizantes e pressão; riscos químicos: substâncias compostas ou produtos químicos no geral, gases, vapores, neblina, poeira, fumo; riscos biológicos: bactéria, protozoários, fungos, vírus, bacilos, parasitas; riscos ergonômicos: levantamento e transporte manual de peso, esforço físico intenso, postura inadequada, ritmos excessivos, jornadas de trabalho intensas, repetitividade de movimentos e /ou qualquer fator que possa interferir nas características psicofisiológicas do trabalhador causando sobrecarga psíquica; e como riscos acidentais: ferramentas inadequadas, eletricidade, arranjo físico inapropriado, máquinas e equipamentos sem proteção, animais peçonhentos, probabilidade de explosão ou incêndio, armazenamento inadequado, e outras situações de risco que coloque o trabalhador em situação vulnerável afetando sua integridade, e seu bem estar físico e psíquico (BRASIL, 2011).

Silva (2014) revela que os profissionais do APH estão expostos a inúmeros riscos advindos de seu processo de trabalho, sendo os principais fatores de adoecimento: os acidentes automobilísticos, contaminação por fluídos ou sangue pela constante de manipulação o que por descuido acidental pode causar contaminação, agressões físicas e verbais, à radiação e a ruídos devido a problemas elétricos, falta de material necessário, elevada carga física e estresse, sendo a violência é o fator apontado como o mais preocupante.

De acordo com Schmoeller et al (2011), as condições de trabalho e a carga excessiva de trabalho dos profissionais que atuam no APH tem gerado risco para a saúde, destacado que a remuneração como inadequada e baixa pelo trabalho. 
Id on Line Revista Multidisciplinar e de Psicologia

Id on Line Multidisciplinary and Psycology Journal

Atuar fora do ambiente hospitalar, levando em conta o grau de complexidade das funções executadas, as responsabilidades do conhecimento técnico e cientifico, o ritmo acelerado, as condições inapropriadas de trabalho e o cenário imprevisível, são condições que podem potencializar os riscos ocupacionais e a ocorrência de alterações físicas e psíquicas, prejudicando seu desempenho profissional.

Alguns participantes do estudo descreveram como riscos ocupacionais:

\begin{abstract}
"Para mim são muitos riscos: manuseio de equipamentos pesados, contato com material infectante, relações interpessoais que envolve trabalho e produtividade, distúrbios do sono, distúrbios alimentares, trabalho sem EPI adequado e péssimas condições de trabalho" (S4).

"Estamos expostos a uma rotina exaustiva, em ambulâncias sem maca, na chuva no sol, atendendo patologias contagiosas. Enfim, somos os primeiros a chegar, sem saber o que vamos encontrar". (S7)
\end{abstract}

Outros fatores de risco relatados pelos participantes do estudo foram a agressão física e psicológica que sofrem tanto dos pacientes, como por seus acompanhantes, e os riscos de adquirir uma doença contagiosa:

\footnotetext{
"A gente sofre risco de exposição à violência e agressão, riscos a doenças contagiosas" (S5).

"Tem risco quanto a integridade física, psicológica, acidente biológico, dentre outras". (S9)
}

Os profissionais do APH ao prestarem atendimento estão expostos em muitos momentos a pacientes psíquicos que apresentam comportamento violento e usuários de álcool e drogas o que torna as agressões como fatores de risco. As agressões físicas são mais comuns em comunidades perigosas, portanto, o local e o tipo de ocorrência oferece a equipe riscos eminentes de morte e agressões, essas situações geram nos profissionais de APH sentimentos de medo, ansiedade e frustração (DE SOUZA, 2015).

$\mathrm{O}$ profissional de $\mathrm{APH}$, na abordagem inicial do paciente, devido à falta de um diagnostica prévio corre o risco de exposição acidentalmente com diversas doenças infecciosas como meningite meningocócica, tuberculose, e gripe, por exemplo (SOUZA; SOUSA; COSTA, 2014).

Dentro desta categoria analítica, todos os profissionais de enfermagem de APH entrevistados afirmaram ter conhecimento a respeito do conceito de riscos ocupacionais e dos 
Id on Line Revista Multidisciplinar e de Psicologia

Id on Line Multidisciplinary and Psycology Journal

riscos envolvidos em sua atuação profissional, destaca-se a seguinte declaração que relaciona as atividades desenvolvidas diariamente aos riscos expostos:

\begin{abstract}
"Riscos físicos- queda, agressão física e verbal, ruídos de sirene, situações de tempo (chuva, exposição ao sol), acidentes automobilísticos, queda de material dentro da viatura durante o deslocamento como: cardioversor, bomba de infusão, maleta de medicamentos. Riscos químicos e biológicos: exposição com gases "oxigênio" material de higienização da viatura, exposição à poeira, bactérias, vírus, secreção, sangue e riscos ergonômicos (rela-cionados ao corpo): postura inadequada, transportar e erguer peso (paciente ou material) de forma errada” (S10).
\end{abstract}

Nessa perspectiva é de extrema importância que o profissional conheça os fatores de risco envolvidos em sua atividade laboral que tem potencial de lhe causar algum agravo ou dano à saúde, bem como as medidas de proteção necessária a fïm de minimizar os riscos de adoecimento no trabalho (SILVA, 2014).

\title{
Categoria 02: As medidas de prevenção de acidentes e adoecimento utilizados no ambiente de trabalho.
}

Ao serem questionados sobre as medidas de prevenção adotadas em relação às atividades de sua categoria profissional, a maioria dos depoentes relataram o uso dos EPIs e o descarte correto de materiais pérfuros cortantes:

\footnotetext{
"Uso de EPI obrigatório, manuseio correto de materiais, descarte correto de perfuro cortante" (S1).

"Utilizamos os EPI,s disponíveis como luva de procedimento, máscara descartável, óculos, botina e macacão” (S11).

"Uso de EPI,s especificos, descartes de materiais perfuro-cortantes e biológicos em locais específicos, limpeza e desinfecção da unidade de trabalho e procurar manter uma postura ergonômica correta, evitar pegar excesso de peso" (S12)

"EPIs e Equipamento Conjugado de Proteção Individual” (S14)
}

A Norma Regulamentadora - NR-6, estabelece como Equipamento de Proteção Individual - EPI, todo dispositivo ou produto, utilizado individualmente pelo trabalhador com a finalidade de promover proteção de riscos suscetíveis à segurança e a saúde no ambiente de trabalho e como Equipamento Conjugado de Proteção Individual, o conjunto de vários 
Id on Line Revista Multidisciplinar e de Psicologia

Id on Line Multidisciplinary and Psycology Journal

dispositivos, que o fabricante relaciona contra um ou mais riscos que possam ocorrer de forma simultânea suscetíveis de ameaça a segurança e a saúde na atividade laboral (BRASIL, 2001).

Portanto os equipamentos de proteção, principalmente, os equipamentos de proteção individual (EPI) são fundamentais para o desenvolvimento do trabalho dos profissionais de saúde do APH garantindo a estes, padrões mínimos de segurança, com a prevenção de riscos ocupacionais.

Segundo a Portaria SIT n. ${ }^{\circ}$ 194, de 07 de dezembro de 2010 da Norma Regulamentadora NR-6, prevê obrigações da empresa em fornecer os EPIs adequado ao risco, gratuitamente e, cabe aos empregados cumprir as determinações do o uso adequado e a responsabilidade pela guarda e conservação. No entanto, os profissionais entrevistados citam que muitas vezes os EPIs não são disponibilizados na quantidade suficiente.

Alguns participantes relatam a falta de EPIs em alguns momentos e a necessidade de improvisar o atendimento, expondo-os aos riscos:

“Utilizar o EPI,s necessários, quando tem a gente usa, mas as vezes falta” (S4)

"Os meios que oferece são muito precários, então temos que a todo momento improvisar, a maioria das vezes. O que resta é esperar que ofereça mais condições de trabalho" (S5).

A falta de materiais de proteção obriga o profissional de APH a improvisar em algumas situações para prestar assistência aos pacientes, muitas vezes em risco iminente de morte, o que contribui para o aumento do índice de riscos ocupacionais gerando um desgaste psicofísico nestes profissionais. Entretanto, muitos profissionais resistem a fazer uso dos EPIs quando fornecidos ou utilizam de maneira inadequada. Alguns profissionais relataram fazer uso apenas de luva de procedimento e máscara, sem uso de óculos de proteção, por exemplo, expondo-se ao risco de respingos de fluidos corpóreos em mucosa ocular, nasal e oral (DE SOUZA, 2015)

Segundo Neves (2011) alguns profissionais de saúde justifica não fazer uso dos equipamentos de proteção pela interferência na habilidade e execução de alguns procedimentos realizados em ritmo acelerado, escolhendo assim por não utilizá-los, o que os torna muito mais vulneráveis a exposição de riscos ocupacionais e acidentes de trabalho.

Os profissionais de APH entrevistados elencaram algumas ações como medidas de prevenção para reduzir o risco de doenças ocupacionais, tais como: 
Id on Line Revista Multidisciplinar e de Psicologia

Id on Line Multidisciplinary and Psycology Journal

\begin{abstract}
"Evitar a sobrecarga de trabalho, evitar movimentos repetitivos, dentre outros. No APH, nosso maior risco são os problemas de coluna, devido ao peso, consequentemente a falta de maca é um dos problemas que agrava a saúde dos trabalhadores, o índice de atestado por conta dessa queixa é significativa, precisamos de uma atenção especial para minimizar esse problema” (S7).

"Uso de EPIs, capacitação anual para prevenção de acidentes. Mas nos não fazemos exames preventivos anualmente, nem acompanhamento com médico do trabalho" $(S 2)$.

"Como medidas de prevenção, podemos está atribuindo e intensificando algumas medidas como: uso de EPIs, treinamento para evitar falhas, apoio da polícia nas ocorrências, fixar equipamentos dentro da unidade, preparo físico e emocional e manutenção preventivas das viaturas" (S9).
\end{abstract}

Segundo De Souza (2015), no que concerne ao levantamento de peso de forma repetitiva, muitas vezes em condições de infraestrutura e postura corporal inadequada constitui as maiores causas de problemas osteomusculares, como as dores lombares, citada pelo entrevistado.

Segundo a Norma Regulamentadora- NR 9 é obrigatório a elaboração e implementação por parte da empresa e intuições a que admitam trabalhadores do Programa de Prevenção de Riscos Ambientais - PPRA que tem como objetivo a preservação da saúde e da integridade dos trabalhadores, incluindo as seguintes etapas: antecipação, reconhecimento, estabelecimento de prioridades e metas, avaliação dos riscos aos quais estão exposto no ambiente de trabalho, bem como as implantação de medidas necessárias de caráter coletivo, acompanhada de treinamento dos trabalhadores para a eliminação, a minimização ou o controle dos riscos ambientais e avaliação de sua eficácia (BRASIL, 1994)

Portanto a educação permanente necessita ser desenvolvida para que o profissional conheça a legislação vigente em saúde do trabalhador e, consequentemente, os riscos ocupacionais a que estão expostos a partir de informações claras e precisas, com questões que envolvam a ampliação de medidas de prevenção e segurança visando à minimização de ocorrências de doenças ocupacionais. 
Id on Line Revista Multidisciplinar e de Psicologia

Id on Line Multidisciplinary and Psycology Journal

\section{Considerações Finais}

No presente estudo foi possível identificar que os profissionais de APH tem conhecimento dos fatores de risco envolvidos no ambiente de trabalho, considerando a sobrecarga de trabalho aliado às falhas na estrutura organizacional, fatores estes determinantes para potencializar os riscos ocupacionais.

Portanto, observa-se a necessidade da educação continuada a respeito de todos os riscos ocupacionais aos quais estão expostos os profissionais de enfermagem no APH e o uso correto dos EPIs, bem como a sensibilização da instituição quanto às dificuldades vivenciadas por esses profissionais relacionadas à sobrecarga de trabalho e recursos materiais. Importante salientar que melhorar as condições de trabalho também minimiza riscos.

\section{Referências}

ALMEIDA, Leilane Grazziela Nascimento; TORRES, Samantha Coelho; DOS SANTOS, Cristiane Magali Freitas. Riscos ocupacionais na atividade dos profissionais de saúde da atenção básica. Revista Enfermagem Contemporânea, v. 1, n. 1, 2012.

ARAUJO, LR de A. MOREIRA,M R; Risco ocupacional enfrentado pela equipe de enfermagem do serviço de atendimento móvel de urgência. V. 2, n.3, 2015.

Brasil. Ministério do Trabalho e Emprego. Normas Regulamentadoras. Disponível em: http:// portal.mte.gov.br/legislacao/normas-regulamentadoras- 1.htm. Acesso em: 10 nov. 2016.

BRASIL. Portaria SIT n. ${ }^{\circ}$ 25, de 15 de outubro de 2001). NR6-Equipamento de proteção Individual. Disponível em: http://www.mte.gov.br/legislacao/normas_regulamentadoras/nr_06.pdf >. Acesso em: 20 de outubro 2016.

BUDEL, Diego GO. Acidente do trabalho: Caracterização, conceito e competência. Direito UNIFACS-Debate Virtual, n. 140, 2012.

DE OLIVEIRA, Queila Borges; DOS SANTOS, Rafaela Sousa; DOS SANTOS, Cristiane Magali Freitas. Acidentes de trabalho na equipe de enfermagem: uma revisão de literatura. Revista Enfermagem Contemporânea, v. 2, n. 1, 2013. 
DE SOUZA, Eudes Rodrigues; DE SOUSA, Alana Tamar Oliveira; COSTA, Isabelle Cristinne Pinto. Riscos ocupacionais no Atendimento Pré-Hospitalar Móvel: produção científica em periódicos online. Revista Brasileira de Ciências da Saúde, v. 18, n. 2, p. 151-156, 2015.

GRAÇA JÚNIOR, C A G G; FREIRE, A K N; DOURADO, G O L; BATISTA, O M A; MADEIRA, M Z A; Riscos ocupacionais a que a equipe de enfermagem está submetida no ambiente hospitalar. In: $61^{\circ}$ Congresso Brasileiro de Enfermagem, 1918, 2009, Fortaleza. Anais eletrônicos...Fortaleza: 2009, p. 1 - 4. Disponível em < http://www.abeneventos.com.br/anais_61cben/files/02465.pdf $>$. Acesso em 30 de jun de 2016.

NEVES, S. A. F. et al. Segurança dos trabalhadores de enfermagem e Fatores determinantes para adesão aos equipamentos de proteção individual. Rev. Latino - Am. Enfermagem 19(2): [08 telas]; mar-abr 2011.

Normas Regulamentadoras. 1994. NR 9 - Programa de prevenção de riscos ambientais. [Internet] Brasília: Ministério do Trabalho e Emprego; 1994. Disponível em http://www.mte.gov.br/legislacao/normas_regulamentadoras/nr_09_at.pdf

OLIVEIRA R.S. Um Estudo Sobre O Risco Ocupacional Em Atendimento Pré-Hospitalar. São Paulo. Trabalho de conclusão em Especialista em Atendimento Pré Hospitalar. 2012.

SCHMOELLER, R. et al. Cargas de trabalho e condições de trabalho da enfermagem: revisão integrativa. Revista Gaúcha de Enfermagem, Porto Alegre, v. 32, n. 2, p. 368-377, 2011.

SILVA, Olvani Martins da et al. Riscos de adoecimento enfrentados pela equipe de enfermagem do SAMU: uma revisão integrativa. Revista de Saúde Pública de Santa Catarina, v. 7, n. 1, p. 107-121, 2014.

SOUSA, F; MACHADO, A; CADETE, E; GRAÇA, L; A que riscos estão expostos os enfermeiros do bloco operatório. Segurança, 25, 19-22, 2004. World Health Organization 2015.Disponívelem<http://apps.who.int/iris/bitstream/10665/170250/1/9789240694439_eng.p df?ua=1\&ua=1>. Acesso em 29 de jun de 2016.

ZAPPAROLI, A dos S; MARZIALE, M H P; Risco ocupacional em unidades de Suporte Básico e Avançado de Vida em Emergências Revista PESQUISA Brasileira de Enfermagem REBEn; Rev Bras Enferm., v. 59., n. 1, 2006.

Como citar este artigo (Formato ABNT):

NASCIMENTO, M.O; ARAÚJO, G.F. Riscos Ocupacionais dos Profissionais de Enfermagem atuantes no SAMU 192. Id on Line Revista Multidisciplinar e de Psicologia, Janeiro de 2017, vol.10, n.33, p.212-223. ISSN: 1981-1179.

Recebido: 01/12/2016

Aceito: $01 / 12 / 2016$ 